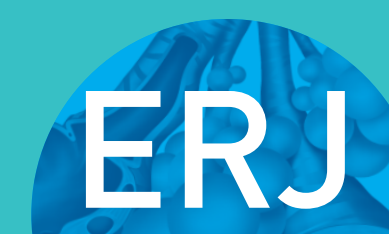

open research
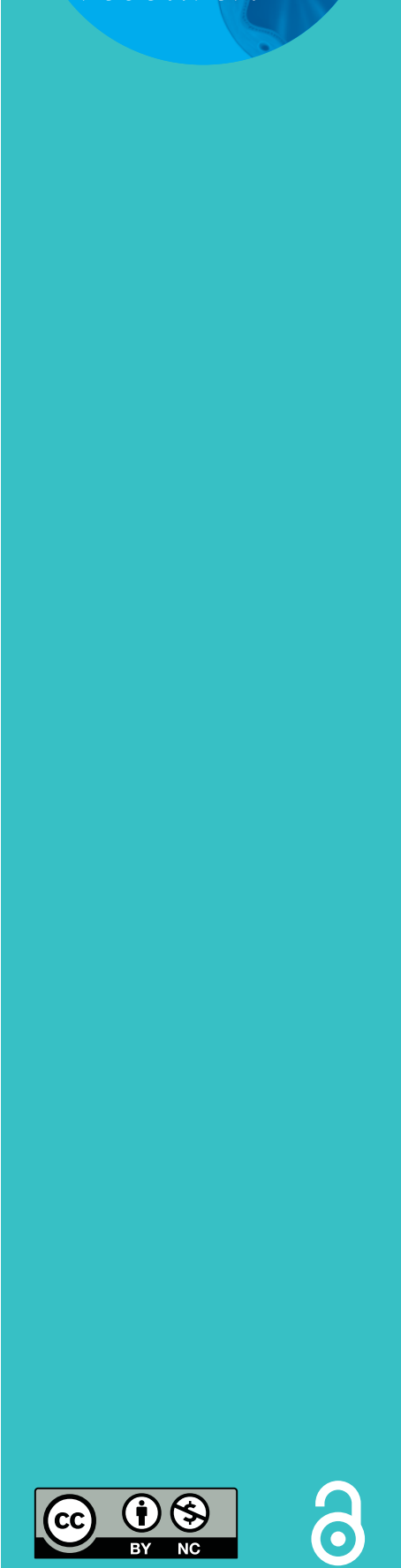

\section{The differing physiology of nitrogen and tracer gas multiple-breath washout techniques}

\author{
Dominic Sandhu (1) ${ }^{1}$, Grant A.D. Ritchie ${ }^{1}$ and Peter A. Robbins (1) ${ }^{2}$
}

Affiliations: ${ }^{1}$ Dept of Chemistry, Physical and Theoretical Chemistry Laboratory, University of Oxford, Oxford, UK. ${ }^{2}$ Dept of Physiology, Anatomy and Genetics, University of Oxford, Oxford, UK.

Correspondence: Peter A. Robbins, Dept of Physiology, Anatomy and Genetics, University of Oxford, Sherrington Building, Parks Road, Oxford OX1 3PT, UK. E-mail: peter.robbinsđadpag.ox.ac.uk

\section{ABSTRACT}

Background: Multiple-breath washout techniques are increasingly used to assess lung function. The principal statistic obtained is the lung clearance index (LCI), but values obtained for LCI using the nitrogen $\left(\mathrm{N}_{2}\right)$-washout technique are higher than those obtained using an exogenous tracer gas such as sulfur hexafluoride. This study explored whether the pure oxygen $\left(\mathrm{O}_{2}\right)$ used for the $\mathrm{N}_{2}$ washout could underlie these higher values.

Methods: A model of a homogenous, reciprocally ventilated acinus was constructed. Perfusion was kept constant, and ventilation adjusted by varying the swept volume during the breathing cycle. The blood supplying the acinus had a standard mixed-venous composition. Carbon dioxide and $\mathrm{O}_{2}$ exchange between the blood and acinar gas proceeded to equilibrium. The model was initialised with either air or air plus tracer gas as the inspirate. Washouts were conducted with pure $\mathrm{O}_{2}$ for the $\mathrm{N}_{2}$ washout or with air for the tracer gas washout.

Results: At normal ventilation/perfusion $\left(V^{\prime} / Q^{\prime}\right)$ ratios, the rate of washout of $\mathrm{N}_{2}$ and exogenous tracer gas was almost indistinguishable. At low $V^{\prime} / Q^{\prime}$, the $\mathrm{N}_{2}$ washout lagged the tracer gas washout. At very low $V^{\prime} / Q^{\prime}, \mathrm{N}_{2}$ became trapped in the acinus. Under low $V^{\prime} / Q^{\prime}$ conditions, breathing pure $\mathrm{O}_{2}$ introduced a marked asymmetry between the inspiratory and expiratory gas flow rates that was not present when breathing air.

Discussion: The use of pure $\mathrm{O}_{2}$ to washout $\mathrm{N}_{2}$ increases $\mathrm{O}_{2}$ uptake in low $V^{\prime} / Q^{\prime}$ units. This generates a background gas flow into the acinus that opposes flow out of the acinus during expiration, and so delays the washout of $\mathrm{N}_{2}$.

@ERSpublications

Differences in lung clearance index between nitrogen and exogenous tracer gas multiple-breath washout tests can be explained by the oxygen used to wash out nitrogen generating convective flows of gas into low ventilation/perfusion units https://bit.ly/310xq0G

Cite this article as: Sandhu D, Ritchie GAD, Robbins PA. The differing physiology of nitrogen and tracer gas multiple-breath washout techniques. ERJ Open Res 2021; 7: 00858-2020 [https://doi.org/ $10.1183 / 23120541.00858-2020]$.

Received: 15 Nov 2020 | Accepted after revision: 2 March 2021

Copyright $\odot$ The authors 2021. This version is distributed under the terms of the Creative Commons Attribution NonCommercial Licence 4.0. For commercial reproduction rights and permissions contact permissions@ersnet.org 


\section{Introduction}

Multiple-breath washout (MBW) techniques are increasingly being used to assess inhomogeneity of lung ventilation as a sensitive marker of early airways disease. The most commonly used index is the lung clearance index (LCI), which gives the number of turnovers of functional residual capacity (FRC) required to reduce the inert gas concentration to $1 / 40$ th of its starting value. However, in a consensus statement from the American Thoracic and European Respiratory Societies [1], it was recognised that there remained considerable variation between different techniques in relation to the values obtained for the LCI.

One common finding across studies is that values for the LCI are higher when measured using nitrogen $\left(\mathrm{N}_{2}\right)$ washout than when measured with an exogenous tracer gas, often sulfur hexafluoride $\left(\mathrm{SF}_{6}\right)$ [2-5]. These studies recognise that the divergence between $\mathrm{N}_{2}$ and $\mathrm{SF}_{6}$ techniques occurs later during the washout profiles, but concede that the underlying mechanism remains unclear. Possible causes suggested include methodological issues, physical differences between the test gases, differences in the pattern of breathing caused by pure oxygen $\left(\mathrm{O}_{2}\right)$ and the role of dissolved $\mathrm{N}_{2}$ in the body.

The present study explores, using a model-based approach, whether the use of pure $\mathrm{O}_{2}$ during a $\mathrm{N}_{2}$ washout could delay the appearance of the $\mathrm{N}_{2}$. Such a suggestion has been made previously in relation to infants with high metabolic rates and low lung volumes [6]. Here, we explore the role of pure $\mathrm{O}_{2}$ with reference to acini with low specific ventilations and ventilation/perfusion $\left(V^{\prime} / Q^{\prime}\right)$ ratios. Such acini would be expected to influence the $\mathrm{N}_{2}$ profile particularly during the later stages of the washout.

\section{Methods}

The model is illustrated in figure 1. The acinus has unit volume at FRC. The blood flow to the acinus $\left(Q^{\prime}{ }_{\text {ac }}\right)$ is set at 2 units $\min ^{-1}$ to reflect that average pulmonary blood flow is approximately twice FRC every minute. For a specific, chosen value $(\mathrm{K})$ for the $V^{\prime} / Q^{\prime}$ ratio for the acinus, the minute ventilation to the acinus $\left(V_{\text {ac }}^{\prime}\right)$ may be calculated directly from the blood flow as $K \times Q^{\prime}{ }_{\text {ac }}$. The acinus is ventilated by varying its volume with a saw-tooth waveform (figure 1). Inspiration lasts $1 \mathrm{~s}$ and expiration lasts $3 \mathrm{~s}$. Thus, to achieve an overall minute ventilation of $V^{\prime}$ ac, the acinus must increase its volume at a rate of $4 \times V^{\prime}{ }_{a c}$ during inspiration, and decrease its volume at a rate of $(4 / 3) \times V^{\prime}$ ac during expiration. The exchange of carbon dioxide $\left(\mathrm{CO}_{2}\right)$ and $\mathrm{O}_{2}$ between the blood flowing through the acinus and the gas in the acinus is assumed to come to equilibrium.

The model was run in 10-ms steps, equivalent to a time interval $(\Delta t)$ of $(1 / 6000) \mathrm{min}$.

First, the volumes for $\mathrm{CO}_{2}$ and $\mathrm{O}_{2}\left(\Delta V_{\mathrm{CO}_{2}}\right.$ and $\Delta V_{\mathrm{O}_{2}}$, respectively) that are released or taken up by the blood during the step are calculated as:

$$
\begin{aligned}
\Delta V_{\mathrm{CO}_{2}} & =Q^{\prime}{ }_{\mathrm{ac}} \times\left(\mathrm{C}_{\mathrm{vCO}_{2}}-\mathrm{C}_{\mathrm{cCO}_{2}}\right) \times \Delta t, \text { and } \\
\Delta V_{\mathrm{O} 2} & =Q^{\prime}{ }_{\mathrm{ac}} \times\left(\mathrm{C}_{\mathrm{cO}_{2}}-\mathrm{C}_{\mathrm{vO}_{2}}\right) \times \Delta t,
\end{aligned}
$$

where $\mathrm{C}_{\mathrm{vCO}}$ and $\mathrm{C}_{\mathrm{vO}}$ are the mixed venous blood gas concentrations for $\mathrm{CO}_{2}$ and $\mathrm{O}_{2}$, respectively, and $\mathrm{C}_{\mathrm{cCO}_{2}}$ and $\mathrm{C}_{\mathrm{cO}_{2}}$ are the end-capillary blood gas concentrations for $\mathrm{CO}_{2}$ and $\mathrm{O}_{2}$, respectively. These

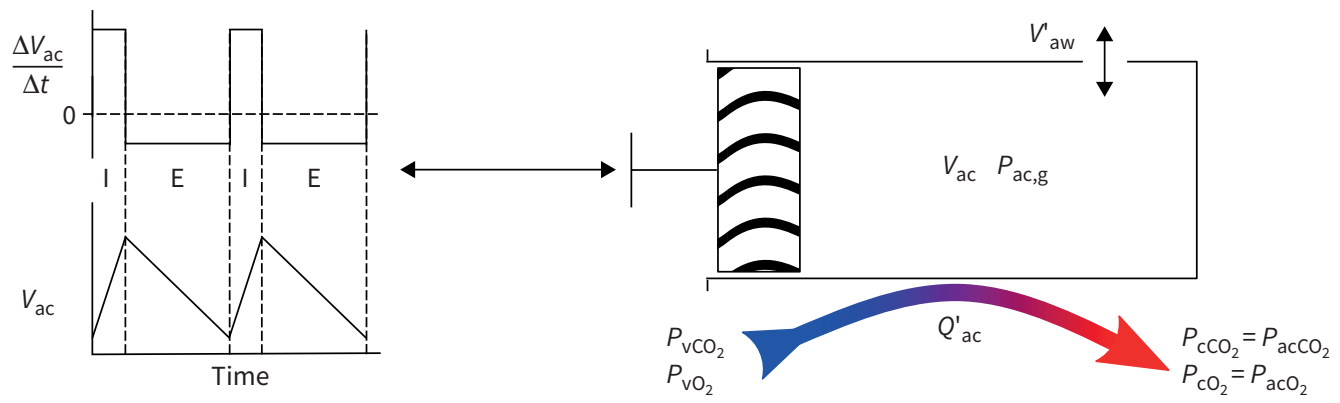

FIGURE 1 Diagram of model acinus. The volume of the acinus $\left(V_{\text {ac }}\right)$ varies in a fixed saw-tooth pattern where the amplitude is set by the value chosen for acinar ventilation/perfusion ratio $\left(V^{\prime}{ }_{\text {ac }} / Q^{\prime}{ }_{\text {ac }}\right)$. The end-capillary partial pressures for carbon dioxide $\left(\mathrm{CO}_{2}\right)$ and oxygen $\left(\mathrm{O}_{2}\right)\left(P_{\mathrm{cCO}_{2}}\right.$ and $P_{\mathrm{CO}_{2}}$, respectively) are equilibrated with the partial pressures in the acinus $\left(P_{\text {acCO}}\right.$ and $P_{\text {acO }}$, respectively). The mixed venous partial pressures for $\mathrm{CO}_{2}$ and $\mathrm{O}_{2}\left(P_{\mathrm{vCO}_{2}}\right.$ and $P_{\mathrm{vO}_{2}}$, respectively) are fixed at $46 \mathrm{mmHg}(6.13 \mathrm{kPa})$ and $40 \mathrm{mmHg}(5.33 \mathrm{kPa})$, respectively. The $\mathrm{O}_{2}$ uptake and $\mathrm{CO}_{2}$ output are calculated from the product of the arterio-venous difference and acinar blood flow $\left(Q^{\prime}{ }_{a c}\right)$. These exchanges, together with the change in $V_{a c}$, are used to determine the overall flow of gas into or out of the airway $\left(V^{\prime}{ }_{\text {aw }}\right)$. $\Delta t$ : time interval; I: inspiration; E: expiration; $P_{\text {ac,g }}$ : partial pressure of a gas in the acinus. 
concentrations are calculated from the corresponding partial pressures $\left(P_{\mathrm{CO}_{2}}\right.$ and $\left.P_{\mathrm{O}_{2}}\right)$ via a model of the blood-gas dissociation curves [7]. For the mixed venous blood, standard values for $P_{\mathrm{CO}_{2}}$ and $P_{\mathrm{O}_{2}}$ were used (46 mmHg/6.13 kPa for $\mathrm{CO}_{2}$ and $40 \mathrm{mmHg} / 5.33 \mathrm{kPa}$ for $\mathrm{O}_{2}$ ). For the end-capillary blood, as $\Delta t$ is short and as complete equilibration is assumed, the $P_{\mathrm{CO}_{2}}$ and $P_{\mathrm{O}_{2}}$ values in the acinus at the start of the $10-\mathrm{ms}$ step were used.

Second, the increment in volume of the acinus over the $10-\mathrm{ms}$ step $\left(\Delta V_{\mathrm{ac}}\right)$ is calculated, either as $4 \times V^{\prime}{ }_{\mathrm{ac}} \times \Delta t$ for inspiration or as $-(4 / 3) \times V^{\prime}{ }_{\mathrm{ac}} \times \Delta t$ for expiration. From these volume changes, the total volume of gas entering from the airway over the 10 -ms step $\left(\Delta V_{\mathrm{aw}}\right)$ can then be calculated as:

$$
\Delta V_{\mathrm{aw}}=\Delta V_{\mathrm{ac}}+\Delta V_{\mathrm{CO}_{2}}-\Delta V_{\mathrm{O}_{2}}
$$

Finally, the amounts of $\mathrm{CO}_{2}, \mathrm{O}_{2}, \mathrm{~N}_{2}$ and exogenous tracer gas (if present) in the acinus can be updated from their amounts entering or leaving from the blood $\left(\mathrm{CO}_{2}\right.$ and $\mathrm{O}_{2}$ only) and the airway. This completes the calculations for the current 10-ms step, and leaves the model with updated volume and gas concentrations in the acinus ready to undertake the next 10 -ms step.

To simulate a $\mathrm{N}_{2}$ washout, the model is first initialised using multiple breath cycles with air as the inspirate until the acinar concentrations are stable between breaths. The $\mathrm{N}_{2}$ washout is then executed by substituting $\mathrm{O}_{2}$ for air in the inspirate. To simulate an exogenous tracer gas washout, the initialisation period is undertaken with a gas that is similar to air, but where $1 \%$ tracer gas has been introduced by replacing an equivalent amount of $\mathrm{N}_{2}$ so that the inspired $\mathrm{O}_{2}$ concentration remains unaltered. The washout is then executed by switching the inspirate to air. Both $\mathrm{N}_{2}$ and exogenous tracer gas are assumed insoluble in blood.

The primary outputs from the model are the acinar gas concentrations at end-expiration (end of each breath cycle), in particular the $\mathrm{N}_{2}$ concentration for the $\mathrm{N}_{2}$ washout and the exogenous tracer gas concentration for the tracer gas washout. The findings are illustrated as semi-log plots, with the $\mathrm{x}$-axis representing the number of whole-lung turnovers, assuming $V^{\prime} / Q^{\prime}$ is equal to 1 for the whole lung. Rather than displaying a large number of discrete points, curves were generated by fitting a spline through the values for the acinar gas concentrations. Secondary outputs included the average rate of gas flow into/out of the acinus, during inspiration and expiration, for each breath, and the relative rates of $\mathrm{N}_{2}$ and tracer gas washout (normalised to their starting concentrations). Again, rather than display large numbers of data points, the results are shown as spline curves fitted through the datapoints.

\section{Results}

The results are shown in figure 2 for a standard $V^{\prime} / Q^{\prime}=1$, a low $V^{\prime} / Q^{\prime}=0.1$ and a very low $V^{\prime} / Q^{\prime}=0.04$.

For a $V^{\prime} / Q^{\prime}=1$, the concentration profiles for $\mathrm{N}_{2}$ and exogenous tracer gas plotted against lung turnover were essentially indistinguishable (figure 2a). The inspiratory and expiratory gas flows $\left(V_{\text {aw }}^{\prime}\right.$ ) each relative to the rate of change of acinar volume $\left(\Delta V_{\mathrm{ac}} / \Delta t\right)$ were slightly above and below one, respectively, reflecting that the respiratory quotient was below unity (figure $2 \mathrm{~b}$ ). The relative rates of elimination of the two gases were always very similar (figure $2 \mathrm{c}$ ).

For a $V^{\prime} / Q^{\prime}=0.1$, the concentration profile against lung turnover declined more steeply for the exogenous tracer gas than for $\mathrm{N}_{2}$ (figure 2a). During the tracer gas washout, the inspiratory and expiratory gas flows remained constant throughout. This was not the case for the $\mathrm{N}_{2}$ washout, where inspiratory flow increased and expiratory flow decreased (figure $2 \mathrm{~b}$ ). This behaviour was generated by the rise in acinar $P_{\mathrm{O}_{2}}$ during the washout increasing the uptake of $\mathrm{O}_{2}$ into the blood by diffusion, and so generating a convective flow of gas from the airway into the acinus. The result was that the rate of elimination was lower for $\mathrm{N}_{2}$ than for the tracer gas during the initial washout, and only reversed when the concentrations of the two gases in the acinus were sufficiently different (figure 2c).

For a $V^{\prime} / Q^{\prime}=0.04$, the results were qualitatively similar to those for a $V^{\prime} / Q^{\prime}=0.1$ except that the concentration profile for $\mathrm{N}_{2}$ plotted against lung turnover plateaued, with almost all the $\mathrm{N}_{2}$ left in the acinus (figure 2a). This occurred because expiratory flow fell below zero, and so gas flow was permanently into the acinus (figure $2 \mathrm{~b}$ ). Thus, most of the $\mathrm{N}_{2}$ was trapped in the acinus and could never wash out through a purely convective process (figure $2 \mathrm{c}$ ).

\section{Discussion}

The modelling provides a key reason why, physiologically, we should not expect MBW tests using $\mathrm{N}_{2}$ and those using exogenous tracer gases to yield the same results, and why the $\mathrm{N}_{2}$ washout will be slower. The essential difference is that exogenous tracer gases are washed out with air, while $\mathrm{N}_{2}$ is washed out with 

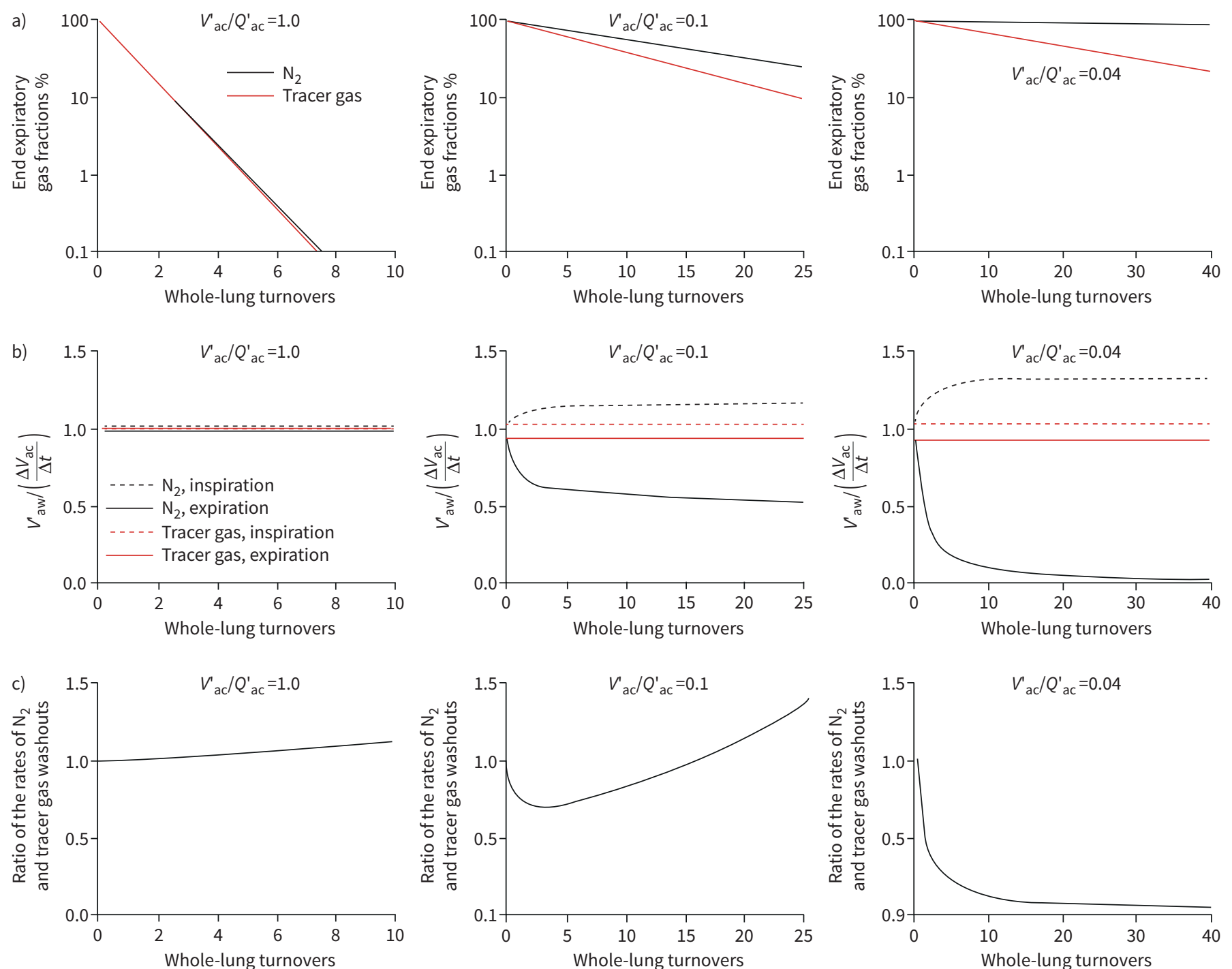

FIGURE 2 Comparison of the effects of low acinar ventilation/perfusion ratio $\left(V^{\prime}{ }^{\prime} / Q^{\prime}{ }^{\prime}\right.$ ac $)$ on rates of nitrogen $\left(N_{2}\right)$ and exogenous tracer gas washout. a) End-expiratory acinar fractions for $N_{2}$ and tracer gas, relative to their starting values at beginning of washout; b) ratio of the flow of gas into or out of the airway ( $V^{\prime}$ aw ${ }^{\prime}$ relative to the rate of change of the volume of the acinus $\left(\Delta V_{a c} \Delta t\right)$ for inspiration and expiration; $\left.c\right)$ ratio for flow of $\mathrm{N}_{2}$ relative to tracer gas out of the acinus during expiration. The flow for each gas species has been normalised to its concentration at start of the washout. Results are presented for values of $V^{\prime}{ }^{\prime} / Q^{\prime}$ ac equal to $1,0.1$ and 0.04 . $x$-axes: number of turnovers of functional residual capacity for whole lung loccur every 30 s). 
pure $\mathrm{O}_{2}$, and the latter influences gas exchange in lung regions of low $V^{\prime} / Q^{\prime}$. In low $V^{\prime} / Q^{\prime}$ units, there is little gas exchange when breathing air and this remains so when an exogenous tracer gas is washed out. However, when washing $\mathrm{N}_{2}$ out with pure $\mathrm{O}_{2}$, the rise in alveolar $P_{\mathrm{O}_{2}}$ generates an uptake of $\mathrm{O}_{2}$ into the blood that then generates an underlying current of gas flow into the lung unit. This current of gas flow opposes the expiratory gas flow generated by breathing, and so reduces the rate at which $\mathrm{N}_{2}$ is washed out of the lung unit.

In addition, the results illustrate that, in the case of very low $V^{\prime} / Q^{\prime}$ units, physiological gas $\left(\mathrm{N}_{2}\right)$ trapping can occur in the lung without physical airways closure. This possibility has been recognised by others previously $[8,9]$. In relation to this, residual $\mathrm{N}_{2}$ in the lung can sometimes be detected at the end of a MBW by a period of forced breathing [10]. Such residual $\mathrm{N}_{2}$ could be arising, in part, through this physiological mechanism as well as by physical gas trapping arising from airways closure. In our simulations, a completely regular breathing pattern was employed. However, variations in expiratory effort occur naturally, and these could result in $\mathrm{N}_{2}$ release during some breaths, but not others. This may explain the difficulty that sometimes occurs identifying the cut off breath when calculating the LCI.

One question that arises naturally from this study is whether acini with low or very low $V^{\prime} / Q^{\prime}$ exist within the lung. Early relatively gross measurements of regional $V^{\prime} / Q^{\prime}$ distribution in healthy humans suggested that values for $V^{\prime} / Q^{\prime}$ may vary between about 0.5 at the base of the lungs to 3 at the apex of the lungs [11]. Following the development of the multiple inert gas elimination technique to assess $V^{\prime} / Q^{\prime}$ distribution, WAGNER et al. [12] concluded that for healthy young people, $>95 \%$ of both the blood flow and the ventilation was directed to units where $V^{\prime} / Q^{\prime}$ was in the range of $0.3-2.1$, but that for some older individuals $V^{\prime} / Q^{\prime}$ values in the range $0.01-0.1$ were observed. What could give rise to such low $V^{\prime} / Q^{\prime}$ units? Pertinent to this question is a recent study by VerLeDEN et al. [13], who used micro-computed tomography to study unused donor lungs and found that, once aged $>30$ years, there was a significant decline in the number of terminal bronchioles. If acini that are lacking terminal bronchioles are not to collapse, then they must be ventilated in some alternative, collateral way. One possible route for this ventilation to occur is via the pores of Kohn. These are considered a high-resistance pathway, potentially with an expiratory resistance that is substantially greater than the inspiratory resistance, and therefore such acini could form the basis of lung tissue with particularly low $V^{\prime} / Q^{\prime}[14,15]$. Indeed, it should be noted that VERLEDEN et al.'s study does not exclude the possibility that there may be acini without terminal bronchioles even in young, healthy lungs. Finally, in airways disease, terminal bronchioles may be lost [16] or simply closed by oedema/inflammation and/or plugged by mucus [17].

A further question that arises is whether very low $V^{\prime} / Q^{\prime}$ units would remain stable when exposed to high $\mathrm{O}_{2}$, or whether they would collapse. This depends on whether or not the low $V^{\prime} / Q^{\prime}$ is associated with a very high inspiratory airways resistance [8]. In such a case, it would be possible for the gas uptake by the blood to exceed the gas flow into the acinus, and therefore collapse would follow. The speed of any such collapse would depend on the $\mathrm{O}_{2}$ and $\mathrm{N}_{2}$ content of the lung unit, and this would be increased if the $\mathrm{N}_{2}$ content of mixed venous blood had been lowered by the period of high $\mathrm{O}_{2}$ breathing so that there was an increased rate of diffusional uptake of $\mathrm{N}_{2}$ into the blood. These matters have been considered in more detail elsewhere [9].

Apart from the influence of pure $\mathrm{O}_{2}$ on the $\mathrm{N}_{2}$ washout, there are other reasons why the washout of $\mathrm{N}_{2}$ may be slower than for exogenous tracer gases. In particular, humans have $\sim 1 \mathrm{~L}$ of dissolved $\mathrm{N}_{2}$ in their tissues, and once the partial pressure of $\mathrm{N}_{2}\left(P_{\mathrm{N}_{2}}\right)$ is lowered in the lung, diffusion of $\mathrm{N}_{2}$ from the blood into the lung will occur $[4,5]$. Taking the solubility of $\mathrm{N}_{2}$ in blood as $1.44 \times 10^{-10} \mathrm{~L}(\mathrm{STPD}) \cdot \mathrm{L}^{-1} \cdot \mathrm{Pa}^{-1}$, an average value of $P_{\mathrm{N}_{2}}$ in the lung of $80 \mathrm{kPa}$ and an average cardiac output of $4 \mathrm{~L} \cdot \mathrm{min}^{-1}$, then the delivery of dissolved $\mathrm{N}_{2}$ via the blood into the lung can be calculated as $46 \mathrm{~mL} \cdot \mathrm{min}^{-1}$. If this were all to be eliminated, then for an alveolar ventilation of $4-5 \mathrm{~L} \cdot \mathrm{min}^{-1}$, it would result in an inspired to end-tidal difference of $\sim 1 \%$. However, the full scale of this effect is unlikely to be sustained for long because, with a lowered $P_{\mathrm{N}_{2}}$ in the arterial blood, the dissolved $\mathrm{N}_{2}$ will wash out of tissues with a high perfusion rate (e.g. kidney, heart) very quickly.

In addition to the physiological reasons for the difference between the rate of washout for $\mathrm{N}_{2}$ and exogenous tracer gases, there are also issues relating to the $\mathrm{N}_{2}$ measurement that could give rise to a falsely slow washout. Guglani et al. [4] identified cross-talk between the $\mathrm{CO}_{2}$ and $\mathrm{N}_{2}$ measurement within an $\mathrm{N}_{2}$ MBW device (Exhalyzer; EcoMedics, Duernten, Switzerland) employed in their study. This resulted in an offset error for the $\mathrm{N}_{2}$ measurement. In seeming contrast to those findings, BAYFIELD et al. [2] found similar LCI values were obtained from this device and from a respiratory mass spectrometer (AMIS 2000; Innovision, Odense, Denmark) when used in tandem during washout studies. However, in healthy volunteers, they also report that a plateau for $\mathrm{N}_{2}$ washout was reached at $\sim 1.3 \%$. This appears high compared with measurements of $\mathrm{N}_{2}$ exchange made using the highly precise technique of laser absorption 
spectroscopy [18]. Finally, it is worth noting that considerable potential exists for cross-talk between $\mathrm{CO}_{2}$ and $\mathrm{N}_{2}$ when using mass spectrometry, as some of the $\mathrm{CO}_{2}$ present may fragment to form carbon monoxide, which has the same molecular mass as $\mathrm{N}_{2}$ at $28 \mathrm{u}$.

Apart from the routines relating to the blood gas dissociation curves, the model used in the present study was extremely simple. This is beneficial in terms of understanding why certain behaviours, such as $\mathrm{N}_{2}$ trapping, can occur. However, it is also a significant limitation as the model does not have the detail necessary to predict with any accuracy the size of the effect. One very important limitation in relation to both $\mathrm{N}_{2}$ and the exogenous tracer gas is that diffusion is constrained just to the acinus where full mixing is assumed. There is no scope for either gas to diffuse into the blood as both are considered insoluble. In addition, there is no scope for back-diffusion into the larger airways. This process is likely to become more important for the very low convective flow rates with which this study has been principally concerned causing the stationary front to migrate to a more proximal position locally within the airways. Therefore, these factors may limit the overall duration for which $\mathrm{N}_{2}$ trapping can persist.

In conclusion, it is already well recognised that MBW tests conducted with $\mathrm{N}_{2}$ as the tracer gas yield slower washouts than those conducted with exogenous tracer gases, and as a consequence that measurements made using the two different techniques are not simply interchangeable. The present, model-based study, illustrates how the use of pure $\mathrm{O}_{2}$ to washout $\mathrm{N}_{2}$ alters gas exchange, and hence slows $\mathrm{N}_{2}$ washout in lung units with particularly low values for $V^{\prime} / Q^{\prime}$. In relation to interpreting parameters relating to the speed of a MBW test, such as the LCI, it is important to recognise that they are simply phenomenological parameterisations of a test result. The results from this study do not invalidate such measurements in the slightest, but by the same token they serve to illustrate further that the interpretation of such test results is complex. Indices such as the LCI are influenced by many factors, and they certainly should not be viewed simply as a measure of the ventilation inhomogeneity within the lung.

Author contributions: P. Robbins conceived project. D. Sandhu wrote the model software and undertook simulations. All authors were involved in drafting and revising the manuscript.

Support statement: This research was funded by the National Institute for Health Research (NIHR) Oxford Biomedical Research Centre and by the EPSRC (EP/T001186/1). The views expressed are those of the authors and not necessarily those of the National Health Service, the NIHR or the Dept of Health. D. Sandhu was supported by a Clarendon (University of Oxford) scholarship. Funding information for this article has been deposited with the Crossref Funder Registry.

Conflict of interest: D. Sandhu reports a Clarendon scholarship (University of Oxford) and personal fees from New College (University of Oxford) during the conduct of the study. G.A.D. Ritchie reports grants from National Institute for Health Research Oxford Biomedical Research Centre, and the Engineering and Physical Sciences Research Council, during the conduct of the study; and has European Patent Application Number 09756339.9 pending and European Patent Number 3314213 issued. P.A. Robbins reports grants from National Institute for Health Research Oxford Biomedical Research Centre, and the Engineering and Physical Sciences Research Council, during the conduct of the study; and has European Patent Application Number 09756339.9 pending and European Patent Number 3314213 issued.

\section{References}

1 Robinson PD, Latzin P, Verbanck S, et al. Consensus statement for inert gas washout measurement using multiple- and single-breath tests. Eur Respir J 2013; 41: 507-522.

2 Bayfield KJ, Horsley A, Alton E, et al. Simultaneous sulfur hexafluoride and nitrogen multiple-breath washout (MBW) to examine inherent differences in MBW outcomes. ERJ Open Res 2019; 5: 00234-2018.

3 Jensen R, Stanojevic S, Gibney K, et al. Multiple breath nitrogen washout: a feasible alternative to mass spectrometry. PLoS One 2013; 8: e56868.

4 Guglani L, Kasi A, Starks M, et al. Difference between $\mathrm{SF}_{6}$ and $\mathrm{N}_{2}$ multiple breath washout kinetics is due to $\mathrm{N}_{2}$ back diffusion and error in $\mathrm{N}_{2}$ offset. J Appl Physiol 2018; 125: 1257-1265.

5 Yammine $\mathrm{S}$, Lenherr $\mathrm{N}$, Nyilas $\mathrm{S}$, et al. Using the same cut-off for sulfur hexafluoride and nitrogen multiple-breath washout may not be appropriate. J Appl Physiol 2015; 119: 1510-1512.

6 Gustafsson PM, Bengtsson L, Lindblad A, et al. The effect of inert gas choice on multiple breath washout in healthy infants: differences in lung function outcomes and breathing pattern. J Appl Physiol 2017; 123: 1545-1554.

7 O'Neill DP, Robbins PA. A mechanistic physicochemical model of carbon dioxide transport in blood. J Appl Physiol 2017; 122: 283-295.

8 Briscoe W, Cree E, Filler J, et al. Lung volume, alveolar ventilation and perfusion interrelationships in chronic pulmonary emphysema. J Appl Physiol 1960; 15: 785-795.

9 Danzker D, Wagner P, West J. Instability of lung units with low Va/Q ratios during $\mathrm{O}_{2}$ breathing. J Appl Physiol 1975; 38: 886-895.

10 Gustafsson PM, Johansson HJ, Dahlbäck GO. Pneumotachographic nitrogen washout method for measurement of the volume of trapped gas in the lungs. Pediatr Pulmonol 1994; 17: 258-268.

11 West JB, Dollery CT. Distribution of blood flow and ventilation-perfusion ratio in the lung, measured with radioactive carbon dioxide. J Appl Physiol 1960; 15: 405-410. 
12 Wagner PD, Laravuso RB, Uhl RR, et al. Continuous distributions of ventilation-perfusion ratios in normal subjects breathing air and $100 \% \mathrm{O}_{2}$. J Clin Invest 1974; 54: 54-68.

13 Verleden SE, Kirby M, Everaerts S, et al. Small airway loss in the physiologically ageing lung: a cross-sectional study in unused donor lungs. Lancet Respir Med 2021; 9: 167-174.

14 Terry PB, Traystman RJ, Newball HH, et al. Collateral ventilation in man. N Engl J Med 1978; 298: 10-15.

15 Morrell NW, Wignall BK, Biggs T, et al. Collateral ventilation and gas exchange in emphysema. Am J Respir Crit Care Med 1994; 150: 635-641.

16 Hogg JC. Pathophysiology of airflow limitation in chronic obstructive pulmonary disease. Lancet 2004; 364: 709-721.

17 West JB. State of the art: ventilation-perfusion relationships. Am Rev Respir Dis 1977; 116: 919-943.

18 Ciaffoni L, O’Neill DP, Couper JH, et al. In-airway molecular flow sensing: a new technology for continuous, noninvasive monitoring of oxygen consumption in critical care. Sci Adv 2016; 2: el600560. 